\title{
Existence and multiple solutions for second-order $p$-Laplacian difference equations
}

Haiping Shi ${ }^{*}$ and Yuanbiao Zhang ${ }^{2}$

\section{*Correspondence:}

shp7971@163.com

${ }^{1}$ Modern Business and

Management Department,

Guangdong Construction

Polytechnic, Guangzhou, 510440,

China

Full list of author information is

available at the end of the article

\begin{abstract}
In this paper, we investigate the solutions of boundary value problems for second-order $p$-Laplacian difference equations. By using the critical point theory, the existence and multiple results are obtained.
\end{abstract}

MSC: 39A10; 47J30; 58E05

Keywords: boundary value problems; $p$-Laplacian; difference equations; critical point theory

\section{Introduction}

In this paper, we consider the following second-order $p$-Laplacian difference equation:

$$
\Delta\left(q_{n} \varphi_{p}\left(\Delta x_{n-1}\right)\right)+f_{n}\left(x_{n}\right)=0, \quad n \in \mathbf{Z}(1, k),
$$

with boundary value conditions

$$
\alpha x_{0}-\beta \Delta x_{0}=0=\gamma x_{k+1}+\sigma \Delta x_{k}
$$

where $\Delta$ is the forward difference operator $\Delta x_{n}=x_{n+1}-x_{n}, \varphi_{p}(s)$ is the $p$-Laplacian operator $\varphi_{p}(s)=|s|^{p-2} s(1<p<\infty), q_{n}$ is real-valued for each $n \in \mathbf{Z}, k$ is a given positive integer, $\alpha, \beta, \gamma$ and $\sigma$ are constants, $f \in C\left(\mathbf{R}^{2}, \mathbf{R}\right)$. Boundary value problem (1.1) with (1.2) contains the following boundary value conditions:

$$
\begin{aligned}
& x_{0}=0, \quad x_{k+1}=0 ; \\
& x_{0}=0, \quad \Delta x_{k}=0 ; \\
& \Delta x_{0}=0, \quad x_{k+1}=0 ;
\end{aligned}
$$

and

$$
\Delta x_{0}=0, \quad \Delta x_{k}=0 .
$$

(c) The Author(s) 2017. This article is distributed under the terms of the Creative Commons Attribution 4.0 International License (http://creativecommons.org/licenses/by/4.0/), which permits unrestricted use, distribution, and reproduction in any medium, provided you give appropriate credit to the original author(s) and the source, provide a link to the Creative Commons license, and indicate if changes were made. 
Eq. (1.1) can be considered as a discrete analogue of the following second-order differential equation:

$$
\left(q(t) \varphi_{p}\left(x^{\prime}\right)\right)^{\prime}+f(t, x(t))=0, \quad t \in \mathbf{R}
$$

Eq. (1.3) includes the following equation:

$$
\left(q(t) \psi\left(x^{\prime}\right)\right)^{\prime}+f(t, x(t))=0, \quad t \in \mathbf{R},
$$

which has arose in the study of fluid dynamics, gas diffusion through porous media and adiabatic reactor [1]. Equations similar in structure to (1.4) arise in the study of homoclinic orbits [2-4] of differential equations [5].

In 2007, Chen and Fang [6] obtained a sufficient condition for the existence of periodic and subharmonic solutions of second-order $p$-Laplacian difference equation

$$
\Delta\left(\varphi_{p}\left(\Delta x_{n-1}\right)\right)+f_{n}\left(x_{n+1}, x_{n}, x_{n-1}\right)=0, \quad n \in \mathbf{Z},
$$

by using the critical point theory. Moreover, Shi et al. [7] investigated the existence and multiplicity results for the Dirichlet boundary value problem (BVP) of (1.4) by using the mountain pass lemma in combination with variational techniques.

Liu et al. [8] in 2013 considered the existence of a nontrivial homoclinic orbit of the following second-order $p$-Laplacian difference equation:

$$
\Delta\left(\varphi_{p}\left(\Delta x_{n-1}\right)\right)-q_{n}\left(\varphi_{p}\left(\Delta x_{n}\right)\right)=f_{n}\left(x_{n+1}, x_{n}, x_{n-1}\right), \quad n \in \mathbf{Z}
$$

and gave some new results.

By using critical point theory, Chen and Tang [9] established some existence criteria to guarantee that the second-order discrete $p$-Laplacian system

$$
\Delta\left(\varphi_{p}\left(\Delta x_{n-1}\right)\right)-a_{n}\left|x_{n}\right|^{p-2} x_{n}+\nabla W_{n}\left(x_{n}\right)=0, \quad n \in \mathbf{Z},
$$

has at least one homoclinic orbit.

In this paper, we investigate the solutions of boundary value problems (1.1) with (1.2) for a second-order $p$-Laplacian difference equation [6-25]. By using the critical point theory [26-28], the existence and multiple results are obtained. The proof is based on the variational methods and linking theorem. The motivation for the present work stems from the recent papers [22, 29].

Let

$$
F(t, z)=\int_{0}^{z} f(t, s) d s \geq 0
$$

and

$$
q_{\min }=\min \left\{q_{n}: n \in \mathbf{Z}(1, k+1)\right\}, \quad q_{\max }=\max \left\{q_{n}: n \in \mathbf{Z}(1, k+1)\right\} .
$$

Let $B_{\rho}$ denote the open ball in $E$ about 0 of radius $\rho$, and let $\partial B_{\rho}$ denote its boundary. 
This paper is divided into four parts. First of all, Section 2 states some basic notations and presents variational structure. Second, in Section 3, we shall state and prove our main results by using the variational methods. Finally, in Section 4, we shall give an example to illustrate the applicability of the main results.

\section{Variational structure}

Our main tool is the critical point theory. We shall establish a suitable variational structure to study the existence of boundary value problem (1.1) with (1.2). At first, we shall state some basic notations which will be used in the proofs of our main results.

Let $\mathbf{R}^{k}$ be a real Euclidean space with dimension $k$. On the one hand, we define the inner product on $\mathbf{R}^{k}$ as follows:

$$
\langle x, y\rangle=\sum_{j=1}^{k} x_{j} y_{j}, \quad \forall x, y \in \mathbf{R}^{k}
$$

by which the norm $\|\cdot\|$ can be induced by

$$
\|x\|=\left(\sum_{j=1}^{k} x_{j}^{2}\right)^{\frac{1}{2}}, \quad \forall x \in \mathbf{R}^{k}
$$

On the other hand, we define the norm $\|\cdot\|_{s}$ on $\mathbf{R}^{k}$ as follows:

$$
\|x\|_{s}=\left(\sum_{j=1}^{k}\left|x_{j}\right|^{s}\right)^{\frac{1}{s}}
$$

for all $x \in \mathbf{R}^{k}$ and $s>1$.

Since $\|x\|_{s}$ and $\|x\|_{2}$ are equivalent, there exist constants $k_{1}, k_{2}$ such that $k_{2} \geq k_{1}>0$, and

$$
k_{1}\|x\|_{2} \leq\|x\|_{s} \leq k_{2}\|x\|_{2}, \quad \forall x \in \mathbf{R}^{k} .
$$

For boundary value problem (1.1) with (1.2), consider the functional $J$ defined on $\mathbf{R}^{k}$ as follows:

$$
J(x)=\frac{1}{p} \sum_{n=1}^{k+1} q_{n}\left|\Delta x_{n-1}\right|^{p}-\sum_{n=1}^{k} F_{n}\left(x_{n}\right)+\varphi_{p}\left(\frac{\gamma}{\sigma}\right) \frac{q_{k+1}^{p} x_{k+1}^{p}}{p}+\varphi_{p}\left(\frac{\alpha}{\beta}\right) \frac{q_{1}^{p} x_{0}^{p}}{p},
$$

where

$$
x=\left\{x_{n}\right\}_{n=1}^{k}=\left(x_{1}, x_{2}, \ldots, x_{k}\right)^{*}, \quad \alpha x_{0}-\beta \Delta x_{0}=0=\gamma x_{k+1}+\sigma \Delta x_{k} .
$$

It is easy to see that $J \in C^{1}\left(\mathbf{R}^{k}, \mathbf{R}\right)$ and, for any $x=\left\{x_{n}\right\}_{n=1}^{k}=\left(x_{1}, x_{2}, \ldots, x_{k}\right)^{*}$, by using $\alpha x_{0}-\beta \Delta x_{0}=0=\gamma x_{k+1}+\sigma \Delta x_{k}$ and the summation by parts

$$
\sum_{n=1}^{k} y_{n} \Delta x_{n-1}=y_{k} x_{k}-y_{1} x_{0}-\sum_{n=1}^{k} \Delta y_{n} x_{n}
$$


we can compute the partial derivative as

$$
\frac{\partial J}{\partial x_{n}}=-\Delta\left(q_{n} \varphi_{p}\left(\Delta u_{n-1}\right)\right)-f_{n}\left(x_{n}\right), \quad \forall n \in \mathbf{Z}(1, k) .
$$

Thus, $x$ is a critical point of $J$ on $\mathbf{R}^{k}$ if and only if

$$
\Delta\left(q_{n} \varphi_{p}\left(\Delta u_{n-1}\right)\right)+f_{n}\left(x_{n}\right)=0, \quad \forall n \in \mathbf{Z}(1, k)
$$

We reduce the existence of boundary value problem (1.1) with (1.2) to the existence of critical points of $J$ on $\mathbf{R}^{k}$. That is, the functional $J$ is just the variational framework of boundary value problem (1.1) with (1.2).

Denote

$$
W=\left\{\left(x_{1}, x_{2}, \ldots, x_{k}\right)^{*} \in \mathbf{R}^{k} \mid x_{n}=c, c \in \mathbf{R}, n \in \mathbf{Z}(1, k)\right\},
$$

and let $V$ be the direct orthogonal complement of $\mathbf{R}^{k}$ to $W$, i.e., $\mathbf{R}^{k}=V \oplus W$.

Let

$$
M=\left(\begin{array}{cccccc}
1 & -1 & 0 & \ldots & 0 & 0 \\
-1 & 2 & -1 & \ldots & 0 & 0 \\
0 & -1 & 2 & \ldots & 0 & 0 \\
\ldots & \ldots & \ldots & \ldots & \ldots & \ldots \\
0 & 0 & 0 & \ldots & 2 & -1 \\
0 & 0 & 0 & \ldots & -1 & 1
\end{array}\right)
$$

be a $(k+1) \times(k+1)$ matrix.

It is easy to see that 0 is an eigenvalue of $M$ and $\xi=\frac{1}{\sqrt{k+1}}(1,1, \ldots, 1)^{*} \in \mathbf{R}^{k+1}$ is an eigenvector of $P$ corresponding to 0 . Let $\lambda_{1}, \lambda_{2}, \ldots, \lambda_{k}$ be the other eigenvalues of $M$. Applying matrix theory, it is obvious that $\lambda_{j}>0$ for all $j \in \mathbf{Z}(1, k)$.

Let

$$
\begin{aligned}
& \lambda_{\max }=\max \left\{\lambda_{j} \mid j=1,2, \ldots, k\right\}, \\
& \lambda_{\min }=\min \left\{\lambda_{j} \mid j=1,2, \ldots, k\right\} .
\end{aligned}
$$

For convenience, we identify $x \in \mathbf{R}^{k}$ with $x=\left(x_{1}, x_{2}, \ldots, x_{k}\right)^{*}$.

\section{Main results}

In this section, we shall state and prove our main results by using the variational methods.

Theorem 3.1 Suppose that the following assumptions are satisfied:

$\left(B_{1}\right) \alpha \geq 0, \beta>0, \gamma \geq 0$ and $\sigma>0$;

$\left(q_{1}\right)$ For any $n \in \mathbf{Z}(1, k+1), q_{n}>0$;

$\left(F_{1}\right)$ There exist constants $c_{1}>0, c_{2}>0$ and $\tau>p$ such that

$$
F(t, y) \geq c_{1}|y|^{\tau}-c_{2}, \quad \forall(t, y) \in \mathbf{R}^{2}
$$

Then boundary value problem (1.1) with (1.2) possesses at least one solution. 
Proof For any $x=\left(x_{1}, x_{2}, \ldots, x_{k}\right)^{*} \in \mathbf{R}^{k}$, combining with $\left(F_{1}\right)$, it is easy to see that

$$
\begin{aligned}
J(x)= & \frac{1}{p} \sum_{n=1}^{k+1} q_{n}\left|\Delta x_{n-1}\right|^{p}-\sum_{n=1}^{k} F_{n}\left(x_{n}\right)+\varphi_{p}\left(\frac{\gamma}{\sigma}\right) \frac{q_{k+1} x_{k+1}^{p}}{p}+\varphi_{p}\left(\frac{\alpha}{\beta}\right) \frac{q_{1} x_{0}^{p}}{p} \\
\leq & \frac{q_{\max } 2^{p}}{p} \sum_{n=1}^{k+1}\left(\left|x_{n}\right|^{p}+\left|x_{n-1}\right|^{p}\right)-c_{1} \sum_{n=1}^{k}\left|x_{n}\right|^{\tau}+c_{2} k+\varphi_{p}\left(\frac{\gamma}{\sigma}\right) \frac{q_{\max }}{p}\|x\|_{p}^{p} \\
& +\varphi_{p}\left(\frac{\alpha}{\beta}\right) \frac{q_{\max }}{p}\|x\|_{p}^{p} \\
\leq & \frac{3 q_{\max } 2^{p}}{p}\|x\|_{p}^{p}-c_{1} k_{2}^{\tau}\|x\|_{\tau}^{\tau}+c_{2} k+\varphi_{p}\left(\frac{\gamma}{\sigma}\right) \frac{q_{\max }}{p}\|x\|_{p}^{p}+\varphi_{p}\left(\frac{\alpha}{\beta}\right) \frac{q_{\max }}{p}\|x\|_{p}^{p} \\
\leq & {\left[\frac{3 q_{\max } 2^{p}}{p}+\varphi_{p}\left(\frac{\gamma}{\sigma}\right) \frac{q_{\max }}{p}+\varphi_{p}\left(\frac{\alpha}{\beta}\right) \frac{q_{\max }}{p}\right] k_{2}^{p}\|x\|^{p}-c_{1} k_{1}^{\tau}\|x\|^{\tau}+c_{2} k \rightarrow-\infty }
\end{aligned}
$$

as $\|x\| \rightarrow+\infty$. By the continuity of $J(x)$, we have from the above inequality that there exist upper bounds of values of functional $J$. Classical calculus shows that $J$ attains its maximal value at some point which is just the critical point of $J$, and the result follows. The proof of Theorem 3.1 is finished.

Theorem 3.2 Suppose that the following hypotheses are satisfied:

$\left(B_{2}\right) \alpha=0, \beta>0, \gamma=0$ and $\sigma>0$;

$\left(q_{2}\right)$ For any $n \in \mathbf{Z}(1, k), q_{n}>0$;

$\left(F_{2}\right)$ There exist constants $\eta_{1}>0, a_{1} \in\left(0, \frac{q_{\min } \lambda_{\min }^{\frac{p}{2}}}{p}\left(\frac{k_{1}}{k_{2}}\right)^{p}\right)$ such that

$$
F(t, y) \leq a_{1}|y|^{p}, \quad \forall|y| \leq \eta_{1}
$$

$\left(F_{3}\right)$ There exist constants $\eta_{2}>0, a_{2} \in\left(\frac{\lambda_{\max }^{\frac{p}{2}} q_{\max }}{p}\left(\frac{k_{2}}{k_{1}}\right)^{p},+\infty\right), a_{3}>0$ such that

$$
F(t, y) \geq a_{2}|y|^{p}-a_{3}, \quad \forall|y| \geq \eta_{2}
$$

where $\lambda_{\min }$ and $\lambda_{\max }$ are constants which can be referred to in (2.4) and (2.5).

Then BVP (1.1) with (1.2) possesses at least two nontrivial solutions.

Remark 3.1 By $\left(F_{3}\right)$ it is easy to see that there exists a constant $a_{4}>0$ such that

$\left(F_{3}^{\prime}\right) F(t, y) \geq a_{2}|y|^{p}-a_{4}, \forall(t, y) \in \mathbf{R}^{2}$.

Lemma 3.1 (Linking theorem $[27,28]$ ) Let E be a real Banach space, $E=E_{1} \oplus E_{2}$, where $E_{1}$ is finite dimensional. Suppose that $J \in C^{1}(E, \mathbf{R})$ satisfies the P.S. condition and

$\left(J_{1}\right)$ There exist constants $a>0$ and $\rho>0$ such that $\left.J\right|_{\partial B_{\rho} \cap E_{2}} \geq a$;

$\left(J_{2}\right)$ There exist $e \in \partial B_{1} \cap E_{2}$ and a constant $R_{0} \geq \rho$ such that $\left.J\right|_{\partial Q} \leq 0$, where $Q=\left(\bar{B}_{R_{0}} \cap\right.$ $\left.E_{1}\right) \oplus\left\{s e \mid 0<s<R_{0}\right\}$.

Then J possesses a critical value $c \geq a$, where

$$
c=\inf _{h \in \Gamma} \sup _{u \in Q} J(h(u))
$$

and $\Gamma=\left\{h \in C(\bar{Q}, E)|h|_{\partial Q}=I\right\}$, where I denotes the identity operator. 
Lemma 3.2 (Clark theorem $[27,28])$ Let $E$ be a real Banach space, $J \in C^{1}(E, \mathbf{R})$, with $J$ being even, bounded from below and satisfying the P.S. condition. Suppose $J(\theta)=0$, there is a set $G \subset E$ such that $G$ is homeomorphic to $S^{n-1}$ ( $n-1$ dimension unit sphere) by an odd map, and $\sup _{G} J<0$. Then $J$ has at least $n$ distinct pairs of nonzero critical points.

Lemma 3.3 Suppose that $\left(B_{2}\right),\left(q_{2}\right),\left(F_{2}\right)$ and $\left(F_{3}\right)$ are satisfied. Then the functional $J$ satisfies the P.S. condition.

Proof It follows from $\left(B_{2}\right)$ that

$$
J(x)=\frac{1}{p} \sum_{n=1}^{k+1} q_{n}\left|\Delta x_{n-1}\right|^{p}-\sum_{n=1}^{k} F_{n}\left(x_{n}\right) .
$$

Let $\left\{x^{(l)}\right\}_{l \in \mathbf{N}} \subset \mathbf{R}^{k}$ be such that $\left\{J\left(x^{(l)}\right)\right\}_{l \in \mathbf{N}}$ is bounded and $J^{\prime}\left(x^{(l)}\right) \rightarrow 0$ as $l \rightarrow \infty$. Then there exists a positive constant $K$ such that

$$
-K \leq\left|J\left(x^{(l)}\right)\right| \leq K, \quad \forall l \in \mathbf{N} .
$$

By $\left(F_{3}^{\prime}\right)$, for any $\left\{x^{(l)}\right\}_{l \in \mathbf{N}} \subset \mathbf{R}^{k}$,

$$
\begin{aligned}
-K & \leq J\left(x^{(l)}\right)=\frac{1}{p} \sum_{n=1}^{k+1} q_{n}\left|\Delta x_{n-1}^{(n)}\right|^{p}-\sum_{n=1}^{k} F_{n}\left(x_{n}^{(l)}\right) \\
& \leq \frac{q_{\max } k_{2}^{p}}{p}\left[\sum_{n=1}^{k+1}\left(\Delta x_{n-1}^{(l)}\right)^{2}\right]^{\frac{p}{2}}-\sum_{n=1}^{k}\left[a_{2}\left|x_{n}^{(l)}\right|^{p}-a_{4}\right] \\
& \leq \frac{q_{\max } k_{2}^{p}}{p}\left\|x^{(l)}\right\|^{p}-a_{2} k_{1}^{p}\left\|x^{(l)}\right\|^{p}+a_{4} k \\
& =\left(\frac{q_{\max } k_{2}^{p} \lambda_{\max }^{\frac{p}{2}}}{p}-a_{2} k_{1}^{p}\right)\left\|x^{(l)}\right\|^{p}+a_{4} k .
\end{aligned}
$$

Thus, we have

$$
\left(\frac{q_{\max } k_{2}^{p} \lambda_{\max }^{\frac{p}{2}}}{p}-a_{2} k_{1}^{p}\right)\left\|x^{(l)}\right\|^{p} \leq K+a_{4} k
$$

From $a_{2} \in\left(\frac{\lambda_{\max }^{\frac{p}{2}} q_{\max }}{p}\left(\frac{k_{2}}{k_{1}}\right)^{p},+\infty\right)$ and the above inequality, it is easy to see that $\left\{x^{(l)}\right\}_{l \in \mathbf{N}}$ is a bounded sequence in $\mathbf{R}^{k}$. Therefore, $\left\{x^{(l)}\right\}_{l \in \mathbf{N}}$ possesses a convergent subsequence in $\mathbf{R}^{k}$ and the result follows. Lemma 3.3 is proved.

Proof of Theorem 3.2 By Lemma 3.3, we have that $J$ is bounded from above on $\mathbf{R}^{k}$. Let $c_{0}=\sup _{x \in \mathbf{R}^{k}} J(x)$. It comes from the proof of Lemma 3.3 that

$$
\lim _{\|x\| \rightarrow+\infty} J(x)=-\infty
$$

This implies that $-J(x)$ is coercive. From the continuity of $J(x)$, there exists $\bar{x} \in \mathbf{R}^{k}$ such that $J(\bar{x})=c_{0}$. It is easy to see that $\bar{x}$ is a critical point of $J$. 
For any $x \in V,\|x\| \leq \rho$, it follows from $\left(F_{2}\right)$ that

$$
\begin{aligned}
J(x) & =\frac{1}{p} \sum_{n=1}^{k+1} q_{n}\left|\Delta x_{n-1}\right|^{p}-\sum_{n=1}^{k} F_{n}\left(x_{n}\right) \\
& \geq \frac{q_{\min } k_{1}^{p}}{p}\left[\sum_{n=1}^{k+1}\left(\Delta x_{n-1}\right)^{2}\right]^{\frac{p}{2}}-a_{1} \sum_{n=1}^{k}\left|x_{n}\right|^{p} \\
& \geq \frac{q_{\min } k_{1}^{p} \lambda_{\min }^{\frac{p}{2}}}{p}\|x\|^{p}-a_{1} k_{2}^{p}\|x\|^{p} \\
& =\left(\frac{q_{\min } k_{1}^{p} \lambda_{\min }^{\frac{p}{2}}}{p}-a_{1} k_{2}^{p}\right)\|x\|^{p} .
\end{aligned}
$$

Thus, $c_{0}>0$. Taking

$$
a=\left(\frac{q_{\min } k_{1}^{p} \lambda_{\min }^{\frac{p}{2}}}{p}-a_{1} k_{2}^{p}\right) \rho^{p}
$$

we have

$$
J(x) \geq a, \quad \forall x \in V \cap \partial B_{\rho}
$$

This implies that $J$ satisfies the condition $\left(J_{1}\right)$ of the linking theorem.

For all $x \in W$, it comes from $\sum_{n=1}^{k+1}\left|\Delta x_{n-1}\right|^{p}=0$ that

$$
J(x)=\frac{1}{p} \sum_{n=1}^{k+1}\left|\Delta x_{n-1}\right|^{p}-\sum_{n=1}^{k} F_{n}\left(x_{n}\right)=-\sum_{n=1}^{k} F_{n}\left(x_{n}\right) \leq 0 .
$$

Therefore, the critical point $\bar{x}$ of $J$ corresponding to the critical value $c_{0}$ is a nontrivial solution of BVP (1.1) with (1.2).

It follows from Lemma 3.3 that $J$ satisfies the P.S. condition on $\mathbf{R}^{k}$. So it remains to verify the condition $\left(J_{2}\right)$.

Take $e \in \partial B_{1} \cap V$, for any $z \in W$ and $r \in \mathbf{R}$, let $x=r e+z$. Then

$$
\begin{aligned}
J(x) & =\frac{1}{p} \sum_{n=1}^{k+1} q_{n}\left|\Delta\left(r e_{n-1}+z_{n-1}\right)\right|^{p}-\sum_{n=1}^{k} F_{n}\left(r e_{n}+z_{n}\right) \\
& =\frac{1}{p} \sum_{n=1}^{k+1} q_{n}\left|\Delta\left(r e_{n-1}\right)\right|^{p}-\sum_{n=1}^{k}\left(a_{2}\left|r e_{n}+z_{n}\right|^{p}-a_{4}\right) \\
& \leq \frac{q_{\max } k_{2}^{p}}{p}\left(\sum_{n=1}^{k+1}\left|\Delta\left(r e_{n-1}\right)\right|^{2}\right)^{\frac{p}{2}}-a_{2} k_{1}^{p}\left(\sum_{n=1}^{k}\left|r e_{n}+z_{n}\right|^{2}\right)^{\frac{p}{2}}+a_{4} k \\
& \leq \frac{q_{\max } k_{2}^{p} \lambda_{\max }^{\frac{p}{2}}}{p} r^{p}-a_{2} k_{1}^{p} r^{p}-a_{2} k_{1}^{p}\|z\|^{p}+a_{4} k \\
& =\left(\frac{q_{\max } \lambda_{\max }^{\frac{p}{2}} k_{2}^{p}}{p}-a_{2} k_{1}^{p}\right) r^{p}-a_{2} k_{1}^{p}\|z\|^{p}+a_{4} k \\
& \leq-a_{2} k_{1}^{p}\|z\|^{p}+a_{4} k .
\end{aligned}
$$


Therefore, for any $x \in \partial Q$, where $Q=\left(\bar{B}_{K_{1}} \cap W\right) \oplus\left\{r e \mid 0<r<K_{1}\right\}$, there exists a constant $K_{1}>\rho>0$ such that $J(x) \leq 0$. By the linking theorem, $J$ possesses a critical value $c \geq \rho>0$, where $c=\inf _{h \in \Gamma} \sup _{x \in Q} J(h(x))$ and $\Gamma=\left\{h \in C\left(\bar{Q}, \mathbf{R}^{k}\right)|h|_{\partial Q}=I\right\}$.

The rest of the proof is similar to that of [7], Theorem 1.1; for the sake of simplicity, we omit its proof.

Theorem 3.3 Suppose that $\left(B_{2}\right),\left(q_{2}\right),\left(F_{2}\right),\left(F_{3}\right)$ and the following hypothesis are satisfied:

(f) $f(t,-y)=-f(t, y)$ for all $(t, y) \in \mathbf{R}^{2}$.

Then BVP (1.1) with (1.2) possesses at least s distinct pairs of nontrivial solutions, where s is the dimension of $V$ which can be referred to in Section 2.

Remark 3.2 The proof of Theorem 3.3 is similar to that of Theorem 4.4 in [12], and we omit its proof.

\section{An example}

As an application of Theorem 3.2, we give an example to illustrate our main result.

Example 4.1 Consider the BVP

$$
\Delta\left(\varphi_{p}\left(\Delta x_{n-1}\right)\right)+\mu x_{n}^{\mu-1}=0, \quad n \in \mathbf{Z}(1, k)
$$

with boundary value conditions (1.2), where $\mu>p$. We have

$$
q_{n} \equiv 1
$$

and

$$
F_{n}\left(x_{n}\right)=x_{n}^{\mu} .
$$

It is easy to verify that all the conditions of Theorem 3.2 are satisfied and then BVP (4.1) with (1.2) possesses at least two nontrivial solutions.

\section{Acknowledgements}

This project is supported by the National Natural Science Foundation of China (No. 11401121) and the Department of Education of Guangdong Province for Excellent Young College Teacher of Guangdong Province.

\section{Competing interests}

The authors declare that they have no competing interests.

\section{Authors' contributions}

All authors read and approved the final manuscript.

\section{Author details}

'Modern Business and Management Department, Guangdong Construction Polytechnic, Guangzhou, 510440, China.

²Packaging Engineering Institute, Jinan University, Zhuhai, 519070, China.

\section{Publisher's Note}

Springer Nature remains neutral with regard to jurisdictional claims in published maps and institutional affiliations. 


\section{References}

1. Esteban, JR, Vázquez, JL: On the equation of turbulent filtration in one-dimensional porous media. Nonlinear Anal. 10(11), 1303-1325 (1986)

2. Guo, CJ, O'Regan, D, Wang, CJ, Agarwal, RP: Existence of homoclinic orbits of superquadratic second-order Hamiltonian systems. Z. Anal. Anwend. 34(1), 27-41 (2015)

3. Guo, CJ, O'Regan, D, Xu, YT, Agarwal, RP: Existence of homoclinic orbits of a class of second-order differential difference equations. Dyn. Contin. Discrete Impuls. Syst., Ser. B, Appl. Algorithms 20, 675-690 (2013)

4. Guo, CJ, O'Regan, D, Xu, YT, Agarwal, RP: Existence of homoclinic orbits for a singular second-order neutral differential equation. J. Math. Anal. Appl. 366, 550-560 (2011)

5. Pinelas, S, Chipot, M, Došlá, Z: Differential and Difference Equations with Applications: Contributions from the International Conference on Differential and Difference Equations and Applications. Springer, New York (2013)

6. Chen, $\mathrm{P}, \mathrm{Fang}, \mathrm{H}$ : Existence of periodic and subharmonic solutions for second-order $p$-Laplacian difference equations. Adv. Differ. Equ. 2007, 042530 (2007)

7. Shi, HP, Liu, ZZ, Wang, ZG: Dirichlet boundary value problems for second order $p$-Laplacian difference equations. Rend. Ist. Mat. Univ. Trieste 42, 19-29 (2010)

8. Liu, X, Zhang, YB, Shi, HP: Homoclinic orbits and subharmonics for second order $p$-Laplacian difference equations. J. Appl. Math. Comput. 43(1-2), 467-478 (2013)

9. Chen, $\mathrm{P}, \mathrm{Tang}, \mathrm{XH}$ : Existence of homoclinic solutions for the second-order discrete $p$-Laplacian systems. Taiwan. J. Math. 15(5), 2123-2143 (2011)

10. AlSharawi, Z, Cushing, JM, Elaydi, S: Theory and Applications of Difference Equations and Discrete Dynamical Systems. Springer, New York (2014)

11. Chen, $\mathrm{P}, \mathrm{Tang}, \mathrm{XH}$ : Infinitely many homoclinic solutions for the second-order discrete $p$-Laplacian systems. Bull. Belg. Math. Soc. Simon Stevin 20(2), 193-212 (2013)

12. Huang, SH, Zhou, Z: On the nonexistence and existence of solutions for a four-order discrete boundary value problem. Adv. Differ. Equ. 2009, 389624 (2009)

13. Leng, JH: Existence of periodic solutions for a higher order nonlinear difference equation. Electron. J. Differ. Equ. 2016, $134(2016)$

14. Leng, $\mathrm{JH}$ : Periodic and subharmonic solutions for $2 n$ th-order $\phi_{c}$-Laplacian difference equations containing both advance and retardation. Indag. Math. 27(4), 902-913 (2016)

15. Liu, X, Zhang, YB, Shi, HP: Existence and multiple solutions for discrete Dirichlet boundary value problems via variational methods. Sci. Bull. "Politeh." Univ. Buchar., Ser. A, Appl. Math. Phys. 75(2), 47-56 (2013)

16. Liu, X, Zhang, YB, Zheng, B, Shi, HP: Periodic and subharmonic solutions for second order $p$-Laplacian difference equations. Proc. Indian Acad. Sci. Math. Sci. 121(4), 457-468 (2011)

17. Shi, HP, Liu, X, Zhang, YB: Homoclinic orbits for second order $p$-Laplacian difference equations containing both advance and retardation. Rev. R. Acad. Cienc. Exactas Fís. Nat., Ser. A Mat. 110(1), 65-78 (2016)

18. Shi, HP, Zhang, YB: Standing wave solutions for the discrete nonlinear Schrödinger equations with indefinite sign subquadratic potentials. Appl. Math. Lett. 58, 95-102 (2016)

19. Yang, LW: Existence of homoclinic orbits for fourth-order p-Laplacian difference equations. Indag. Math. 27(3), 879-892 (2016)

20. Yang, LW: Existence theorems of periodic solutions for second-order difference equations containing both advance and retardation. J. Contemp. Math. Anal. 51(2), 58-67 (2016)

21. Zhou, Z, Ma, DF: Multiplicity results of breathers for the discrete nonlinear Schrödinger equations with unbounded potentials. Sci. China Math. 58(4), 781-790 (2015)

22. Zhou, Z, Su, MT: Boundary value problems for $2 n$-order $\phi_{c}$-Laplacian difference equations containing both advance and retardation. Appl. Math. Lett. 41, 7-11 (2015)

23. Zhou, Z, Yu, JS: Homoclinic solutions in periodic nonlinear difference equations with superlinear nonlinearity. Acta Math. Sin. Engl. Ser. 29(9), 1809-1822 (2013)

24. Zhou, Z, Yu, JS: On the existence of homoclinic solutions of a class of discrete nonlinear periodic systems. J. Differ. Equ. 249(5), 1199-1212 (2010)

25. Zhou, Z, Yu, JS, Chen, YM: Homoclinic solutions in periodic difference equations with saturable nonlinearity. Sci. China Math. 54(1), 83-93 (2011)

26. Chen, $\mathrm{P}, \mathrm{He}, \mathrm{XF}$, Tang, XH: Infinitely many solutions for a class of fractional Hamiltonian systems via critical point theory. Math. Methods Appl. Sci. 39(5), 1005-1019 (2016)

27. Mawhin, J, Willem, M: Critical Point Theory and Hamiltonian Systems. Springer, New York (1989)

28. Rabinowitz, PH: Minimax Methods in Critical Point Theory with Applications to Differential Equations. Am. Math. Soc., Providence (1986)

29. Chen, P, Tang, XH: Existence of solutions for a class of second-order $p$-Laplacian systems with impulsive effects. Appl. Math. 59(5), 543-570 (2014) 